\title{
Large Anterior Mediastinal Mass: Intraoperative Transesophageal Echocardiography Guided Evaluation and Management
}

\author{
Kulbhushan Saini ${ }^{*}$, Sandeep Chauhan ${ }^{2}$, Manu Saini ${ }^{3}$ \\ ${ }^{1}$ Department of Anesthesia and Intensive Care, Post Graduate Institute of Medical Education and Research, Chandigarh, India \\ ${ }^{2}$ Department of Cardiac Anesthesia, All India Institute of Medical Sciences, New Delhi, India \\ ${ }^{3}$ Department of Ophthalmology, Advanced Eye Center, Post Graduate Institute of Medical Education and Research, Chandigarh, India \\ Email: ${ }^{\star}$ kulbhushansaini007@gmail.com
}

How to cite this paper: Saini, K., Chauhan, S. and Saini, M. (2021) Large Anterior Mediastinal Mass: Intraoperative Transesophageal Echocardiography Guided Evaluation and Management. World Journal of Cardiovascular Surgery, 11, 69-74.

https://doi.org/10.4236/wjcs.2021.118010

Received: June 17, 2021

Accepted: August 28, 2021

Published: August 31, 2021

Copyright $\odot 2021$ by author(s) and Scientific Research Publishing Inc. This work is licensed under the Creative Commons Attribution International License (CC BY 4.0).

http://creativecommons.org/licenses/by/4.0/

\begin{abstract}
The authors report a case of Large Anterior mediastinal mass in which Transoesophageal Echocardiography (TEE) revealed a large mass that was compressing the right atrium, SVC, henceforth, TEE helped in guiding aspiration and surgical resection of the cystic mass. Therefore, through this case report, authors emphasize the importance of performing intraoperative real-time TEE in the mediastinal mass. This imaging technique helps in visualizing the external compression of cardio-vascular structure and aid in their management, obviating the possibility of large vascular structures from being injured by the surgeon during intraoperative manipulation.
\end{abstract}

\section{Keywords}

Transoesophageal Echocardiography, Superior Vena Cava, Trans Thoracic Echocardiography, Computed Tomography, Right Atrium, Inferior Vena Cava

\section{Introduction}

Anterior Mediastinal masses possess special deliberation for anaesthesiologists, as they can be associated with hemodynamic compromise from extrinsic vascular and cardiac compression, can potentially cause neurological problems (raised intracranial pressure, headache) from obstructed venous drainage in superior vena cava, or respiratory compromise from airway obstruction and lung atelectasia [1]. 
Transoesophageal Echocardiography (TEE) may provides very useful information [2] by continuous monitoring during the intraoperative period, when impacted large mass is being treated manually over the cardiac chambers and vascular structures.

In our case, TEE-guided aspiration and surgical resection of the cystic mass was performed after confirmation of large mass compressing the right atrium \& SVC on TEE, which was not detected on preoperative Transthoracic Echocardiography (TTE). The "written" informed consent has been obtained from the patient for submission of the Clinical Case Report for potential publication.

\section{Case Report}

A 45-year-old, 44-kg female was admitted to our institution with a 2-month complained of right-sided chest pain in anterior location that increases with deep inspiration, cough, and history of approximately $5 \mathrm{Kg}$ weight loss.

During evaluation chest radiography demonstrated a right-sided chest mass with right-sided pleural effusion (Figure 1).

Further Diagnostic evaluation included a chest computed tomography (CT) scan and a transthoracic echocardiogram. The CT scan results confirmed a large anterior mediastinal cystic lesion measuring $13.6 \mathrm{~cm} \times 12 \mathrm{~cm} \times 11 \mathrm{~cm}$ (craniocaudal $\times$ transverse $\times$ anteroposterior) abutting closely the mediastinum. The superomedial part of the large cyst was located in the anterior mediastinum just posterior to the sternum. Medially the lesion was abutting the mediastinum and displacing it towards the left side. The mass was also compressing the right lower zone of the adjacent lung and collapsing it (Figure 2).

Transthoracic echocardiogram confirmed normal biventricular and valvular function and did not commented on compression of cardiovascular structures by surrounding large mass. After taking the written informed consent, the patient was shifted in the operating room for surgical resection of the mass with cardiopulmonary bypass facility available standby in the operation theatre.

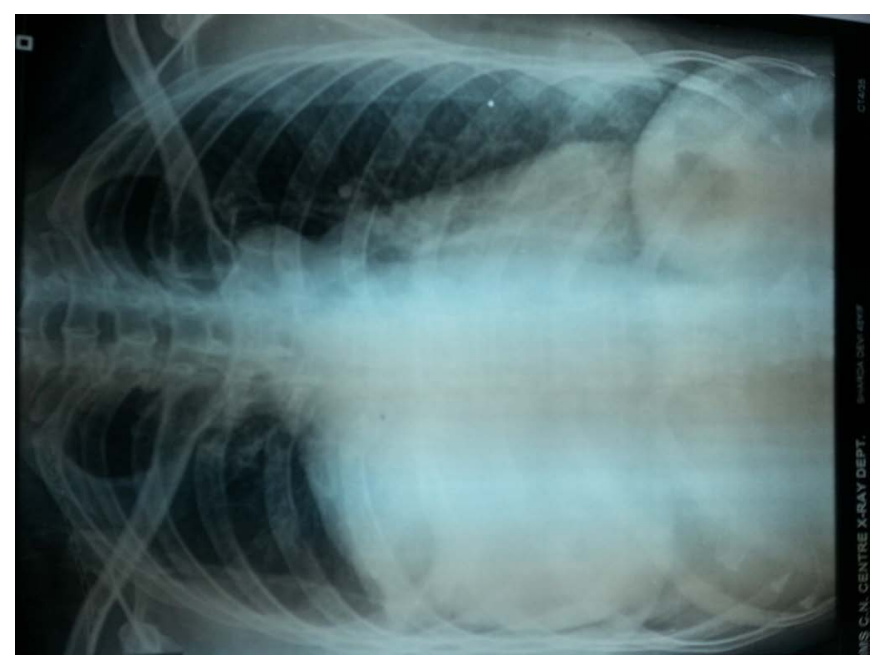

Figure 1. Chest X-ray showing homogenous opacity in right side. 


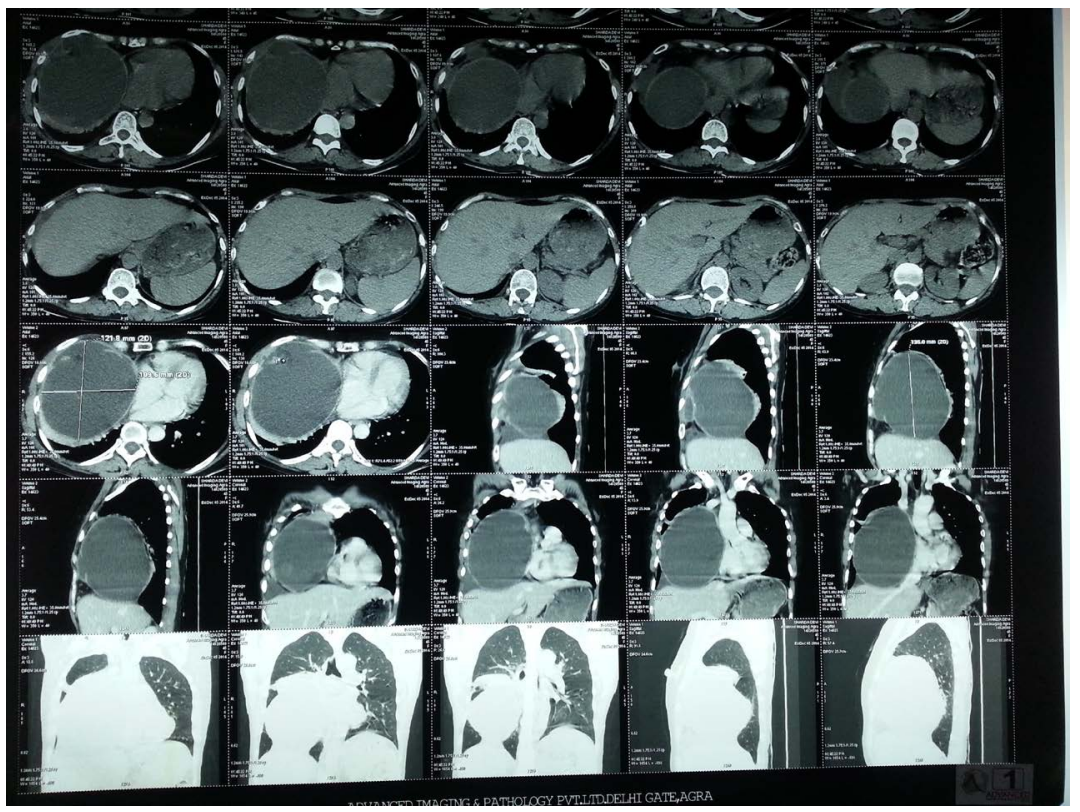

Figure 2. CT Scan showing anterior mediastinal mass in right side.

After induction of general anaesthesia, safely median sternotomy was performed but cardiac structures were not visible from the eyes because the whole mediastinum was filled with pus (Figure 3).

At that time TEE was a real saviour for us as it demonstrated real-time cardiac structures underlying the mass. Afterwards, surgeon aspirated the pus under real time TEE guidance that aspirating needle doesn't injure the underlying vessels or cardiac chambers.

Although after sternotomy cardiac structures were not seen but at that time TEE examination, revealed a large mass compressing the SVC and Right Atrium (RA) (Figure 4 and Figure 5).

Inferior Vena Cava (IVC) was dilated because due to right atrium compression RA pressure was increased (Figure 6).

Initially guided aspiration of the mass was performed cautiously to prevent inadvertent damage to the SVC or right atrium (Figure 7).

After aspiration, clear cystic cavities were seen in an empty sac of mass and finally, surgeons resected the whole mass (Figure 8).

\section{Discussion}

Intraoperatively, patients with anterior mediastinal masses may develop catastrophic superior vena cava obstruction, hemodynamic instability due to cardiac compromise caused by extrinsic compression. CT scan images showed that the massive tumor present in this patient had occupied almost the whole of the mediastinum.

Her cardiac examination was normal with TEE but TEE helped immensely, intraoperative by visualizing extravascular compression by mass on large vessels and cardiac chambers while mass manipulation was done during resection. 


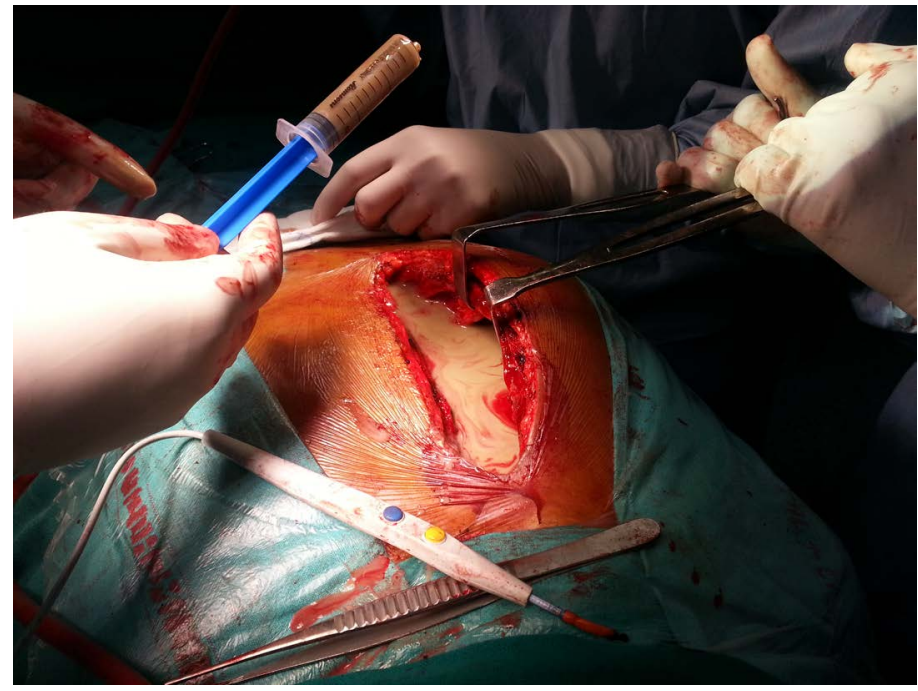

Figure 3. Pus filled chest cavity after sternotomy, Cardiac structures are not visible.

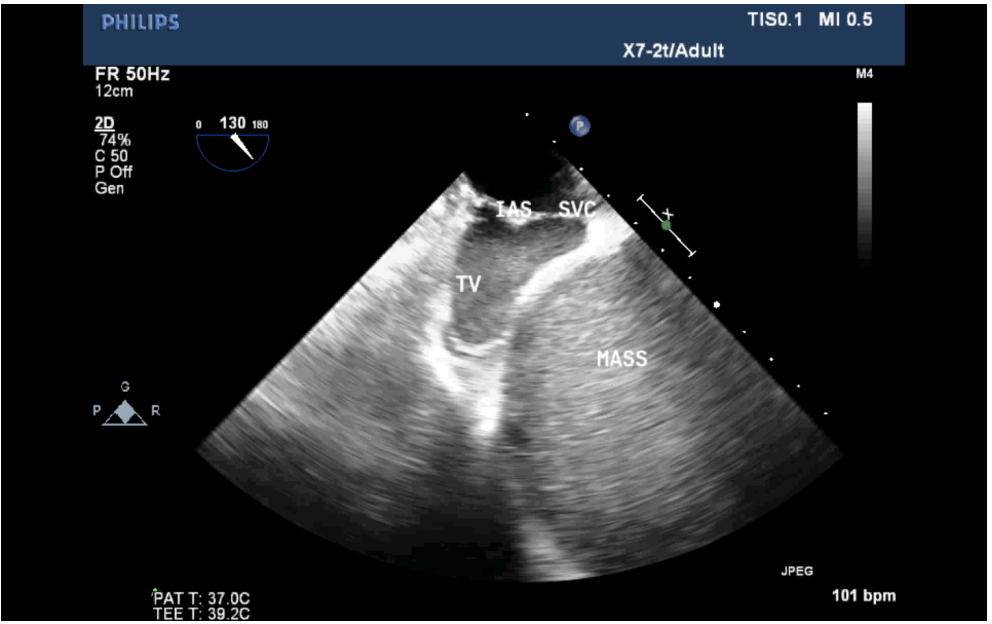

Figure 4. Midesophageal bicaval view showing hypoechoic mass surrounded by hyperechoic wall compressing the SVC and RA.

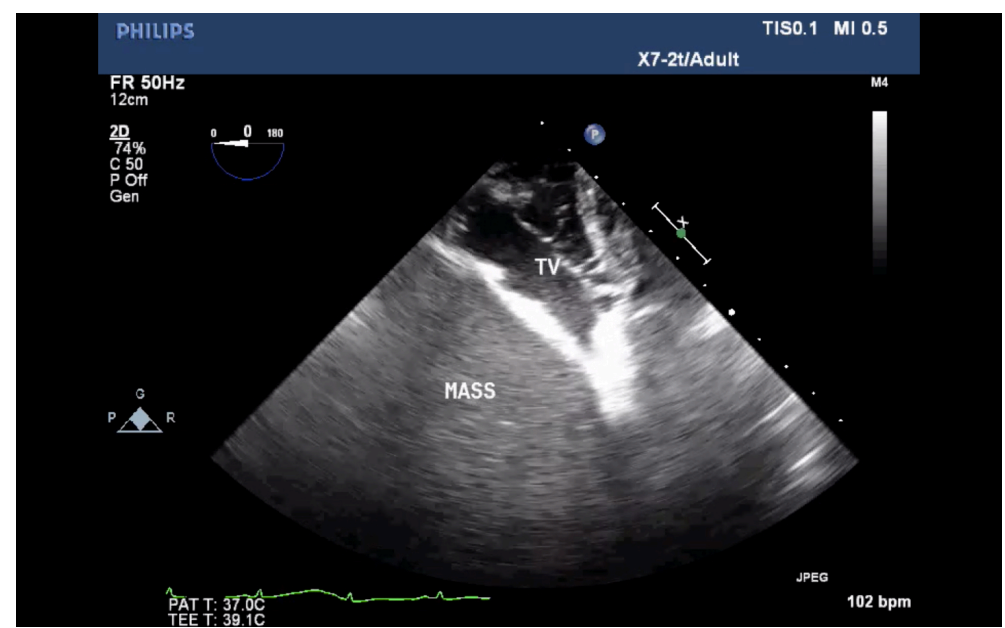

Figure 5. Transgastric modified tricuspid valve view showing homogenous mass compressing RA in which TV is seen. 


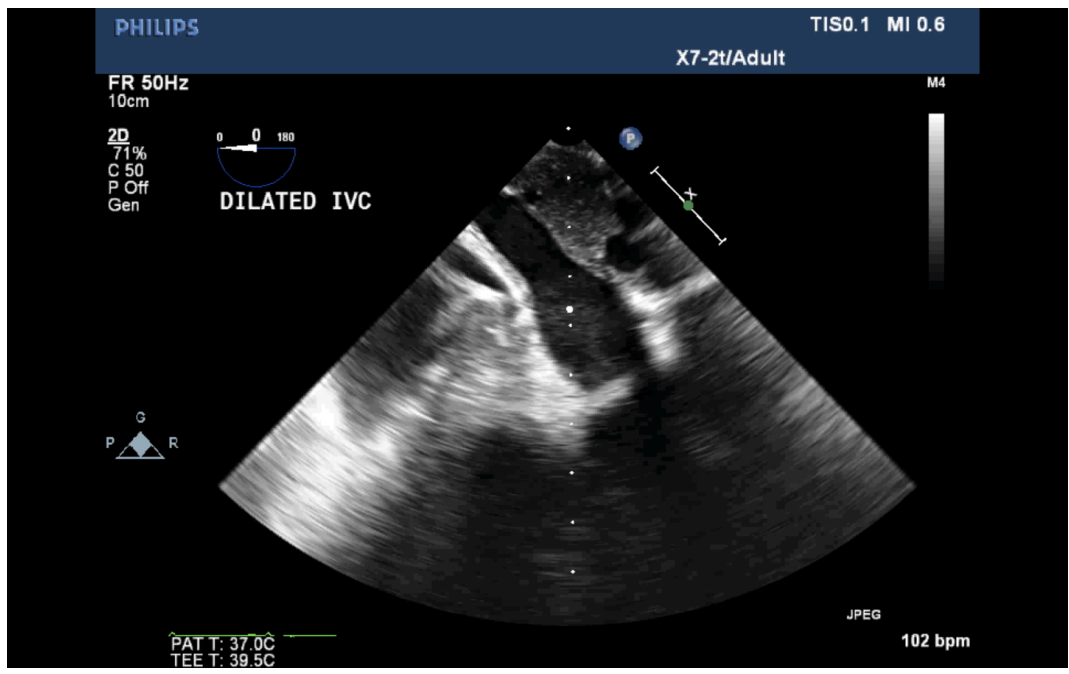

Figure 6. TEE demonstrated concomitant dilated IVC due to increase RA pressure.

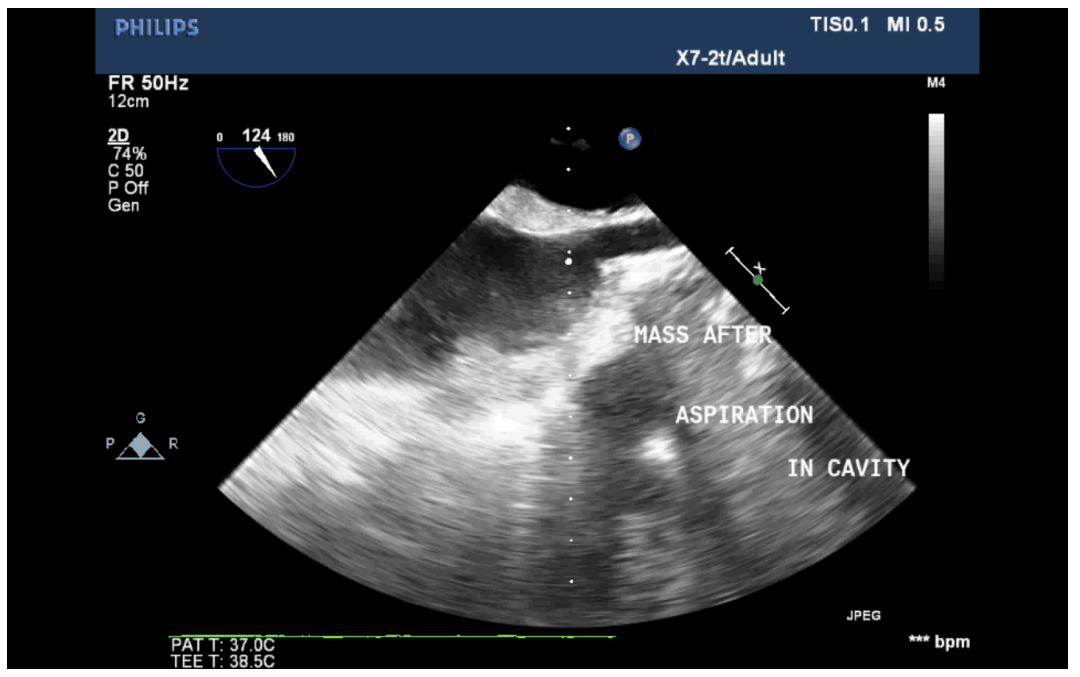

Figure 7. Midesophageal bicaval view showing mass after aspiration.

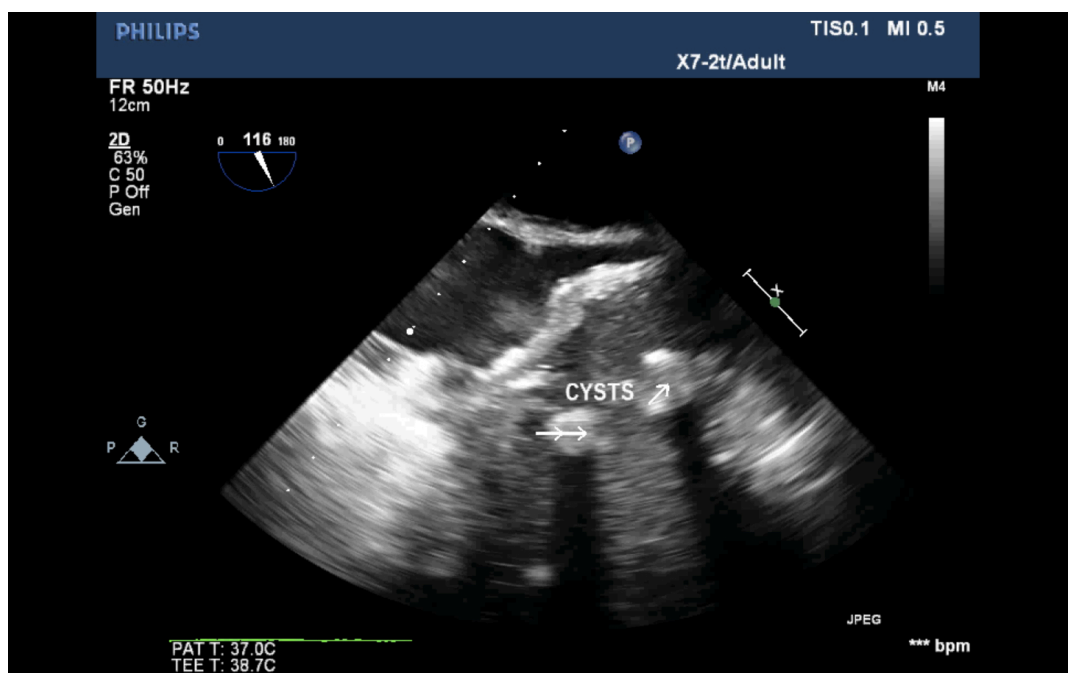

Figure 8. Midesophageal bicaval view showing cystic cavities in mass after aspiration. 
Hemodynamic complications anticipated in such a massive tumor included direct compression over the cardiac chambers, compression of the great vessels [3], and pericardial effusion [4]. Diastolic filling may be compromised and they may present with rhythm disturbances. The presence of cardiovascular compression has been proved to be definitely associated with adverse outcomes in the intraoperative period [4]. Compression of superior vena cava may severely diminish venous return to the Right atrium and compromise preload of the heart.

Preoperative Transthoracic Echocardiography [5] is useful in detecting mediastinal masses but here intraoperative TEE helps in assessing the degree of great vessels and myocardial compression apart from global cardiac function.

In this case, based on the real-time TEE findings by anaesthesiologists, the surgical team planned the aspiration of the cyst under real-time TEE guidance while preserving the adjacent vascular structures from being injured by aspirating needle.

\section{Conclusion}

TEE-guided intervention intraoperatively, serves as the novel and safeguard approach in large mediastinal mass surgery.

\section{Conflicts of Interest}

None.

\section{References}

[1] Dubey, P.K. and Tripathi, N. (2018) Anesthetic Considerations in a Patient with Large Anterior Mediastinal Mass. Journal of Cardiothoracic and Vascular Anesthesia, 33, 1073-1075. https://doi.org/10.1053/j.jvca.2018.03.023

[2] Daniel, T. and Alex, S. (2006) Transesophageal Echocardiography for the Intraoperative Evaluation of a Large Anterior Mediastinal Mass. Anesthesia \& Analgesia, 103, 578-579. https://doi.org/10.1213/01.ane.0000226095.59926.94

[3] Halpern, S., Chatten, J., Meadows, A.T., Byrd, R. and Lange, B. (1983) Anterior Mediastinal Masses: Anesthesia Hazards and Other Problems. The Journal of Pediatrics, 102, 407-410. https://doi.org/10.1016/S0022-3476(83)80664-7

[4] Béchard, P., Létourneau, L., Lacasse, Y., Côté, D. and Bussières, J.S. (2004) Perioperative Cardiorespiratory Complications in Adults with Mediastinal Mass: Incidence and Risk Factors. Anesthesiology, 100, 826-834. https://doi.org/10.1097/00000542-200404000-00012

[5] D’Cruz, I.A., Feghali, N. and Gross, C.M. (1994) Echocardiographic Manifestations of Mediastinal Masses Compressing or Encroaching on the Heart. Echocardiography, 11, 523-533. https://doi.org/10.1111/j.1540-8175.1994.tb01093.x 